\title{
Evolution of State-Dependent Risk Preferences
}

\author{
PATRICK ROOS, J. RYAN CARR, and DANA S. NAU \\ University of Maryland
}

Researchers have invested much effort in constructing models of the state-dependent (sometimes
risk-averse and sometimes risk-prone) nature of human decision making. An important open
question is how state-dependent risk behavior can arise and remain prominent in populations. We
believe that one part of the answer is the interplay between risk-taking and sequentiality of choices
in populations subject to evolutionary population dynamics. To support this hypothesis, we provide
simulation and analytical results for evolutionary lottery games, including results on evolutionary
stability. We consider a parameterized class of imitation dynamics in which the parameter $0 \leq \alpha \leq 1$
yields the replicator dynamic with $\alpha=1$ and the imitate-the-better dynamic with $\alpha=0$. Our results
demonstrate that for every population dynamic in this class except for the replicator dynamic, the
interplay between risk-taking and sequentiality of choices allows state-dependent risk behavior to
have an evolutionary advantage over expected-value maximization.

Categories and Subject Descriptors: I.2.11 [Artificial Intelligence]: Distributed Artificial Intelligence-Multiagent systems; I.6.3 [Simulation and Modeling]: Applications; J.4 [Social and Behavioral Sciences]

General Terms: Human Factors, Theory, Experimentation

Additional Key Words and Phrases: Evolutionary games, population dynamics, risk, decision theory

ACM Reference Format:

Roos, P., Carr, J. R., and Nau, D. S. 2010. Evolution of state-dependent risk preferences. ACM Trans. Intell. Syst. Technol. 1, 1, Article 6 (October 2010), 21 pages.

DOI $=10.1145 / 1858948.1858954$ http://doi.acm.org/10.1145/1858948.1858954

\section{INTRODUCTION}

Empirical evidence shows that human decision making, rather than conforming to the decision-theoretic notion of expected-value maximization, is

This work was supported in part by AFOSR grant FA95500610405 and ARO grant W911NF0920072. The opinions expressed in this article are those of the authors and do not necessarily reflect the opinions of the funders.

Author's address: P. Roos, J. R. Carr, and D. S. Nau, Laboratory for Computational Cultural Dynamics, Department of Computer Science, Institute for Advanced Computer Studies, and Institute for Systems Research, University of Maryland, College Park, MD 20742; email: \{roos, carr2, nau\}@cs.umd.edu.

Permission to make digital or hard copies of part or all of this work for personal or classroom use is granted without fee provided that copies are not made or distributed for profit or commercial advantage and that copies show this notice on the first page or initial screen of a display along with the full citation. Copyrights for components of this work owned by others than ACM must be honored. Abstracting with credit is permitted. To copy otherwise, to republish, to post on servers, to redistribute to lists, or to use any component of this work in other works requires prior specific permission and/or a fee. Permissions may be requested from Publications Dept., ACM, Inc., 2 Penn Plaza, Suite 701, New York, NY 10121-0701 USA, fax +1 (212) 869-0481, or permissions@acm.org. (C) 2010 ACM 2157-6904/2010/10-ART6 $\$ 10.00$

DOI 10.1145/1858948.1858954 http://doi.acm.org/10.1145/1858948.1858954

ACM Transactions on Intelligent Systems and Technology, Vol. 1, No. 1, Article 6, Publication date: October 2010. 
state-dependent: the decisions are sometimes risk-averse and sometimes riskseeking, depending on the decision maker's circumstances. Much effort has been invested in describing and modeling such behavior, but these efforts have largely lacked an explicit investigation of what evolutionary pressures might have influenced the behavior's spread. Thus an important open question is why state-dependent risk behavior is so prevalent.

In this article, we use tools from evolutionary game theory to investigate how agents' risk behavior relates to different population dynamics (i.e., rules governing changes in the number of agents of each kind). In particular, we are interested in imitation dynamics, which model cultural evolution as a product of social learning by imitation. The best-known imitation dynamics are the replicator dynamic and the imitate-the-better dynamic, but there are many others.

We consider a parameterized class of imitation dynamics in which the parameter $0 \leq \alpha \leq 1$ yields the replicator dynamic with $\alpha=1$ and the imitatethe-better dynamic with $\alpha=0$. Our study includes (1) a detailed analysis of how different imitation dynamics can affect risk behavior when agents make sequential choices, and (2) simulations, using several different imitation dynamics, of simple evolutionary lottery games in which agents make sequential choices among lotteries that have equal expected value but different risks. Our results demonstrate that for every population dynamic in this class except for the replicator dynamic, the interplay between risk-taking and sequentiality of choices allows state-dependent risk behavior to have an evolutionary advantage over expected-value maximization.

Our study provides a starting point for further investigation of how population dynamics influence risk behavior in evolutionary game environments. We anticipate that state-dependent risk behavior will outperform expected-valuemaximizing strategies in a wide variety of evolutionary game environments involving sequential choices of different risks.

The remainder of this article is organized as follows. Section 2 provides a brief background on human decision making under risk and evolutionary game theory. Section 3 introduces our evolutionary game model, including the lottery game we use to study risk behavior and the imitation dynamics we are interested in. Section 4 provides theoretical analysis and predictions of how we expect different risk behavior to perform in different environments. Section 5 presents our experimental simulations and results. Finally, Section 6 provides concluding remarks.

\section{BACKGROUND}

\subsection{Human Decision Making Under Risk}

Human decision making under risk is the subject of much research effort in the social sciences. In most of the existing literature on models of human decision making under risk, the construction of such models is approached primarily through the analysis of a decision maker's choices among lotteries that have different payoff distributions, and thus potentially different risks. Under the 
most traditional model of decision making, expected utility theory, a rational agent's preferences can be modeled by assigning to each possible outcome a number called the outcome's utility; and a rational choice is one that maximizes the expected utility of the outcomes [von Neumann and Morgenstern 1944]. Empirical evidence of human decision making under risk shows that humans are sometimes risk-averse, sometimes risk-seeking, and even behave in ways that systematically violate the axioms of expected utility [Kahneman and Tversky 1979]. Expected utility theory can account for different attitudes towards risk through certain von Neumann-Morgenstern utility functions (e.g., Friedman and Savage [1948]). Such risk propensities can differ greatly from simple expected-value considerations on prospective outcomes.

Researchers have invested much effort in constructing utility functions that appropriately model human decision making using risk using the expectedutility model (e.g., Friedman and Savage [1948]; Arrow [1971]; Rabin [2000]). Related efforts in economics have aimed to describe the preferences of humans over inter-temporal lotteries, recognizing the effects of temporally successive lotteries on risk preferences [Epstein and Zin 1989, 1991]. Other studies define utility functions that take into account inter-personal or population comparisons [Abel 1990]. Yet other researchers have constructed alternative descriptive theories of decision making that claim to correspond more closely to how humans make decisions involving risk. Among the most popular of these models are prospect theory [Kahneman and Tversky 1979; Tversky and Kahneman 1992], regret theory [Loomes and Sugden 1982], and SP/A (SecurityPotential/Aspiration) theory [Lopes 1987, 1990; Lopes and Oden 1999]. One advantage of these models is that they more explicitly or perhaps more naturally model some of the mechanics involved in human decision-making processes. For example, state-dependent attitudes toward risk are modeled in prospect theory by using a reference point with respect to which prospective outcomes can be interpreted as potential gains or losses, and are modeled in SP/A theory by including an aspiration level as an additional decision criterion in decisions involving risk. A common theme of both Prospect theory and SP/A theory is that agents are risk-averse when they have done well relative to some reference point and risk-seeking when they have not done well relative to the reference point.

\subsection{Evolutionary Game Theory and Risk Behavior}

Evolutionary game theory has been used to study and explain a great variety of social and cultural phenomena. Examples of such phenomena studied through evolutionary games include altruism [Axelrod and Hamilton 1981; Riolo et al. 2001; Bowles and Gintis 2004], trust [Fang et al. 2002; Kimbrough 2005], fairness [Binmore 1998], empathy [Page and Nowak 2002], and social learning [Schotter and Sopher 2003]. The social science literature is filled with examples of empirical studies showing that humans violate the strong rationality assumptions of classical game theory. Evolutionary game theory is thus an attractive framework under which to model human behavior in such domains because the assumption of perfectly rational agents is not required. 
Furthermore, most game-theoretic work in economics, the focus of our evolutionary-game-theoretic approach is not to define internal preferences and whether or not agents satisfy these. Rather, the focus is to evaluate the preferences according to their evolutionary fitness (which is external).

Evolutionary game theory studies a population of agents making choices according to an assigned strategy in a game that models a situation of interest. After agents have played the game, they reproduce into the next generation according to a reproduction function or population dynamic that, generally speaking, increases the frequency of the types of agents that were successful in the current generation. To study risk behavior in this framework, we can model the situation of interest as a game in which agents are faced with choices among lotteries of different risks. As described in Alexander [2009], these lotteries dispense resources that are considered to be an objective quantity of which (1) agents always want more than less and (2) interpersonal comparisons are meaningful. The reproduction function defining the dynamics of strategies in the population then acts directly on these resources.

\subsection{Imitation Dynamics}

Imitation dynamics are a class of population dynamics commonly used to model the evolution of behaviors in societies [Hofbauer and Sigmund 1998; Nowak and May 1992; Huberman and Glance 1993; Nowak et al. 1994; Eguluz et al. 2005]. The general framework for imitation dynamics is stated by Hofbauer and Sigmund [1998] as follows.

We shall suppose that occasionally a player is picked out of the population and afforded the opportunity to change his strategy. $\mathrm{He}$ samples another player at random, and adopts his strategy with a certain probability.

In what follows, we will call these players the "observer" and the "observed agent," respectively.

Important theoretical studies have been done of two specific imitation dynamics. One of these is the replicator dynamic [Taylor and Jonker 1978; Schlag 1998, 1999; Hofbauer and Sigmund 1998; Gintis 2000], in which the probability that the observer adopts the strategy of the observed agent is proportional to how much more successful the observer was than the observed. The other is the imitate-the-better dynamic [Blume 1993; Vega-Redondo 1997; Szabó and T "oke 1998; Riolo et al. 2001; Hauert and Szabo 2005; Dawid 2007; Traulsen and Hauert 2009; Traulsen et al. 2007], ${ }^{1}$ in which the observer always adopts the observed agent's strategy if it was more successful than the observer's strategy. ${ }^{2}$

Several experimental studies investigating social learning through imitation between humans have found experimental support for Vega-Redondo's model [Huck et al. 1997, 2000; Offerman et al. 2002]. Experiments on human imitation reported by Apesteguia et al. [2007] indicate that the difference in observed

\footnotetext{
${ }^{1}$ The imitate-the-better dynamic is sometimes called tournament selection [Riolo et al. 2001].

${ }^{2}$ Vega-Redondo [1997] generalizes the imitate-the-better dynamic by allowing the observer to observe a collection of agents and adopt the strategy of the most successful agent in $C$.
} 
payoff to an agent's own payoff does affect imitation (the higher the difference, the more likely imitation occurs). This is more in line with Schlag's model. Due to this evidence for both imitation models, in this article we explore a parameterized range of imitation dynamics based on a definition in Hofbauer and Sigmund [1998] that includes the replicator dynamic, the imitate-thebetter dynamic, and a spectrum of other dynamics in between these two.

\section{EVOLUTIONARY GAME MODEL}

In previous work we have proposed the sequential lottery game, a class of games that we use to investigate risk behavior under evolutionary pressures [Roos and Nau 2009, 2010b]. In this section we describe this sequential lottery game and the particular range of imitation dynamics under which we explore the evolution of risk behavior.

\subsection{Sequential Lottery Game}

We consider a game in which agents acquire payoffs dispensed by lotteries. In each generation, each agent must make a sequence of $n$ choices where each choice is between two lotteries: a safe lottery, whose payoff is always 4, and a risky lottery, which one can win (a payoff of 8) with probability $p$, or lose (a payoff of 0 ) with probability $1-p .^{3}$ Note that if $p=0.5$, both lotteries have expected value 4 .

Our population consists of agents that follow strategies chosen from the set $\mathcal{S}=\left\{s_{1}, \ldots, s_{k}\right\}$ of all possible pure strategies for the sequence of lottery choices. In any generation, a vector $\mathbf{x}=\left(x_{1}, \ldots, x_{k}\right)$ gives the state of the population, where each $x_{i}$ is the proportion of agents in the population using strategy $s_{i}$. We let $\pi(i)$ denote the payoff accumulated in a generation from the $n$ lottery choices by agents of type $i$ (i.e., agents following strategy $s_{i}$ ).

\subsection{Imitation Dynamics in Our Evolutionary Games}

As we discussed in Section 2.3, we want to explore a range of population dynamics that includes the replicator dynamic, imitate-the-better dynamic, and other dynamics intermediate between these two extremes. Hofbauer and Sigmund [1998] give the following parameterized formula for these population dynamics:

$$
x_{i}=x_{i} \sum_{j} x_{j}|\pi(i)-\pi(j)|^{\alpha} \operatorname{sign}(\pi(i)-\pi(j)),
$$

where $x_{i}$ is the current proportion in the population of agents of type $i, x_{i}$ is the change in $x_{i}$ over time, ${ }^{4}$ and $\alpha \geq 0$ is a parameter that determines the particular

\footnotetext{
${ }^{3}$ The choice of payoffs here is arbitrary. The results in this article (see Sections 4 and 5) would be qualitatively similar if we had used any fixed value $v$ for the safe lottery's payoff and $v+\delta$ and $v-\delta$ (for fixed $\delta$ ) for the risky lottery's payoffs.

${ }^{4} \mathrm{As}$ is common in the evolutionary game-theory literature, Hofbauer and Sigmund [1998] approximate the current population as a real-valued function $x_{i}(t)$, where $t$ is the current time, so that $x_{i}=d x_{i} / d t$.
} 
imitation dynamic. Our formulation is based on theirs, but incorporates the following changes.

- We are interested in population dynamics based on payoff comparisons among individuals as in Blume [1993]; Szabó and T'oke [1998], Riolo, Cohen, and Axelrod [2001]; Hauert and Szabo [2005]; Traulsen and Hauert [2009]; Traulsen et al. [2007]; Dawid [2007]. To model payoff comparisons among individuals, we must take into account the stochastic variability in the payoffs to individual agents. We do this by treating $\pi(i)$ and $\pi(j)$ as discrete random variables representing the distributions of payoffs that an agent of type $i$ and an agent of type $j$ receive from their lottery choices.

- The imitate-the-better dynamic and the replicator dynamic correspond to $\alpha=0$ and $\alpha=1$, respectively. Since the imitation dynamics that interest us are these two and and the ones that are intermediate between them, we only consider $0 \leq \alpha \leq 1$.

If we let $r$ and $s$ be any possible payoff values acquired by agents of type $i$ and $j$, and let $p(r, s)$ be the probability of obtaining this pair of values, then our modified version of Eq. (1) is

$$
x_{i}=x_{i} \sum_{j} x_{j} \nabla_{\alpha}(i, j)
$$

where $\nabla_{\alpha}(i, j)=\sum_{r} \sum_{s}|(r-s)|^{\alpha} \operatorname{sign}(r-s) \cdot p(r, s)$ is the switching rate between two agent types $i$ and $j$. This switching rate determines the effect that a pairing for imitation between agents of type $i$ and $j$ has, on average, on type $i$ 's growth rate in the population. For example, if $\nabla_{\alpha}(i, j)$ is positive, a pairing for imitation between agents of type $i$ and $j$ (which happens with nonzero probability if agents of type $i$ and $j$ exist in the population) on average has a positive effect on $i$ agents' growth rate. If this is the case, we say $i$ has an evolutionary advantage over $j$.

\section{THEORETICAL PREDICTIONS}

In the two-choice sequential lottery game, there are six possible pure strategies. These are listed in Table I. ${ }^{5}$

Table II gives each strategy, its possible numeric payoffs, and the probabilities of these payoffs for the case where $p$, the probability of wining the risky lottery, is 0.5 . Note that in this case, all six strategies have the same expected payoff value of 8 , but they have differing probabilities of being above or below 8. For example, $P[\pi(\mathrm{RwS})>8]=0.5$ and $P[\pi(\mathrm{RwS})<8]=0.25$.

In this section, we examine how these strategies will perform against one another for different population dynamics (Sections 4.1, 4.2, and 4.3) and for

\footnotetext{
${ }^{5}$ For simplicity, we have restricted our study to pure strategies. For lottery games like the ones we are considering, the reproductive fitness of a mixed strategy should be intermediate among the pure strategies in the mixed strategy's support, hence the inclusion of mixed strategies should not have a substantial affect on our results. If the agents were interacting in a nonzero-sum game rather than making simple choices among lotteries, then the inclusion of mixed strategies could make a significant difference in the results but such games are beyond the scope of this article.
} 
Table I. All of the Possible Pure Strategies in Our Lottery Game When $n=2$

\begin{tabular}{l|lll}
\hline Strategy & 1st Lottery & \multicolumn{1}{c}{ 2nd Lottery } \\
\hline$S S$ & choose safe & choose safe \\
$R R$ & choose risky & choose risky \\
$S R$ & choose safe & choose risky \\
$R S$ & choose risky & choose safe \\
$R w S$ & choose risky & choose safe if 1st lottery was won, risky otherwise \\
$R w R$ & choose risky & choose risky if 1st lottery was won, safe otherwise \\
\hline
\end{tabular}

Table II. Payoff Distributions of the Six Pure Strategies in the Sequential Lottery Game with $n=2$ and $p=0.5$.

All six strategies have the same expected payoff, 8 , but differing distributions. In this table and others in this section, we use boldface numbers to denote payoff values, and nonboldface to denote probabilities

\begin{tabular}{|c|c|c|c|c|c|c|c|c|c|c|c|c|c|c|}
\hline \multirow[b]{2}{*}{ Payoff $V$} & \multicolumn{3}{|c|}{ RwS } & \multicolumn{3}{|c|}{ RwR } & \multicolumn{2}{|c|}{ SR } & \multicolumn{2}{|c|}{$\mathrm{RS}$} & SS & \multicolumn{3}{|c|}{$\mathrm{RR}$} \\
\hline & 12 & 8 & $\mathbf{0}$ & 16 & 8 & 4 & 12 & 4 & & 4 & 8 & 16 & 8 & 0 \\
\hline rol & .5 & .25 & .25 & .25 & .25 & .5 & .5 & .5 & .5 & .5 & 1 & .25 & .5 & .25 \\
\hline
\end{tabular}

different expected values of the lotteries (Section 4.4), culminating in an evolutionary stability result for the RwS strategy (Section 4.5). Finally, Section 4.6 briefly discusses cases where $n \neq 2$.

\subsection{The Replicator Dynamic}

We now show that under the replicator dynamic $(\alpha=1)$, the switching rate between any two agent types is equal to the difference in their expected payoffs.

Proposition 4.1. Under the population dynamics given by Eq. (2) when $\alpha=1, \nabla_{\alpha}(i, j)$ (the switching rate between any two agent types $i$ and $j$ ) is the difference between the expected payoffs of agents of type $i$ and $j$ (given by the discrete random variables $\pi(i)$ and $\pi(j))$.

Proof. Let $r$ and $s$ be possible payoff values acquired by agents of type $i$ and $j$, and let $p(r, s)$ be the probability of obtaining this pair of values. With $\alpha=1$,

$$
\begin{aligned}
\nabla_{\alpha}(i, j) & =\sum_{r} \sum_{s}|(r-s)|^{\alpha} \operatorname{sign}(r-s) \cdot p(r, s) \\
& =\sum_{r} \sum_{s}(r-s) \cdot p(r, s)=E V(\pi(i)-\pi(j)) .
\end{aligned}
$$

Assuming independence between payoffs, $\nabla_{\alpha}(i, j)=E V(\pi(i)-\pi(j))=E V(\pi(i))$ $-E V(\pi(j))$, and the proposition follows.

Since all strategies have the same expected payoff in this environment, Proposition 4.1 tells us that the switching rate between any two strategies will be 0 . Consequently, all six of the preceding strategies will perform equally well evolutionarily. 
Table III. Payoff Distribution for RwS vs. RR in the Sequential Lottery Game with $n=2$ and $p=0.5$.

Entries in the table show give the probabilities that each pair of payoffs occurs

\begin{tabular}{c|c|l|l|l|}
\multicolumn{1}{c}{} & \multicolumn{3}{c}{ RwS } \\
\cline { 3 - 5 } \multicolumn{1}{c|}{} & $\mathbf{1 2}$ & $\mathbf{8}$ & \multicolumn{1}{c|}{$\mathbf{0}$} \\
\hline \multirow{4}{*}{$\mathrm{16}$} & 0.125 & 0.0625 & 0.0625 \\
\cline { 2 - 5 } & $\mathbf{8}$ & 0.25 & 0.125 & 0.125 \\
\hline & $\mathbf{0}$ & 0.125 & 0.0625 & 0.0625 \\
\hline
\end{tabular}

\subsection{The Imitate-the-Better Dynamic}

If we use the imitate-the-better dynamic ( $\alpha=0)$, then in Eq. (2), only the sign of the payoff difference between two paired agents plays a role in determining the switching rate; the magnitude of the difference is irrelevant. We can compute the switching rate between two strategies by using the probabilities in Table II to calculate the probability of each pair of payoffs occurrig. Table III shows these values for the RwS vs. RR pairing. We can then use these probabilities as the values of $p(r, s)$ in Eq. (2) to calculate the switching rate, as follows.

$$
\begin{aligned}
\nabla_{\alpha}(\mathrm{RwS}, \mathrm{RR})= & \operatorname{sign}(0-0) \cdot 0.0625+\operatorname{sign}(8-0) \cdot 0.0625+\operatorname{sign}(12-0) \cdot 0.125 \\
& +\operatorname{sign}(0-8) \cdot 0.125+\operatorname{sign}(8-8) \cdot 0.125 \\
& +\operatorname{sign}(12-8) \cdot 0.25+\operatorname{sign}(0-16) \cdot 0.0625 \\
& +\operatorname{sign}(8-16) \cdot 0.0625+\operatorname{sign}(12-16) \cdot 0.125 \\
= & 0.0625+0.125-0.125+0.25-0.0625-0.0625-0.125 \\
= & 0.0625 .
\end{aligned}
$$

Using similar calculations, we see that $\nabla_{0}(\mathrm{RwS}, \mathrm{SS})=0.25, \nabla_{0}(\mathrm{RwS}, \mathrm{RwR})=$ 0.0625 , and $\nabla_{0}(\mathrm{RwS}, \mathrm{RS})=\nabla_{0}(\mathrm{RwS}, \mathrm{SR})=0$. This suggests that RwS will be able to consistently win an evolutionary competition against RR, RwR, or SS and remain stable with SR and RS in this environment. The experimental results in Section 5 verify this prediction.

\subsection{Arbitrary $\alpha$}

For $0 \leq \alpha \leq 1$, we can calculate the switching rate in a method similar to the previous section, combining the probabilities from Table II to get the values of $p(r, s)$ for Eq. (2). However, since both the sign and magnitude of the payoff differences are now important, the calculation is slightly more complex. For instance, the switching rate for the RwS vs. RR pairing is now as follows.

$$
\begin{aligned}
\nabla_{\alpha}(\mathrm{RWS}, \mathrm{RR})= & |0-0|^{\alpha} \operatorname{sign}(0-0) \cdot 0.0625+|8-0|^{\alpha} \operatorname{sign}(8-0) \cdot 0.0625 \\
& +|12-0|^{\alpha} \operatorname{sign}(12-0) \cdot 0.125+|0-8|^{\alpha} \operatorname{sign}(0-8) \cdot 0.125 \\
& +|8-8|^{\alpha} \operatorname{sign}(8-8) \cdot 0.125+|12-8|^{\alpha} \operatorname{sign}(12-8) \cdot 0.25 \\
& +|0-16|^{\alpha} \operatorname{sign}(0-16) \cdot 0.0625+|8-16|^{\alpha} \operatorname{sign}(8-16) \cdot 0.0625 \\
& +y|12-16|^{\alpha} \operatorname{sign}(12-16) \cdot 0.125 \\
= & 4^{\alpha}(0.125)+8^{\alpha}(-0.125)+12^{\alpha}(0.125)+16^{\alpha}(-0.0625) .
\end{aligned}
$$




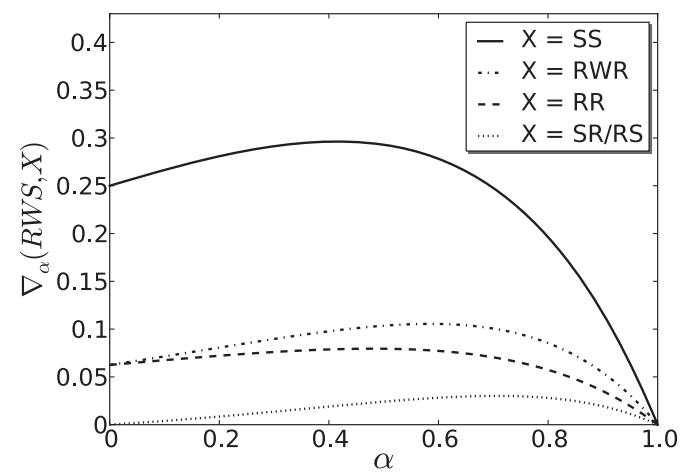

Fig. 1. Switching rate between RwS and each of the other five pure strategies for $0 \leq \alpha \leq 1$. A positive switching rate indicates an evolutionary advantage of RwS against the other strategy (in a population made up of solely the two strategies). We see that RwS has such an advantage over all other strategies when $0<\alpha<1$, and all but SR and RS when $\alpha=0$.

Table IV. Payoff Distributions of the Six Pure Strategies in the Sequential Lottery Game with $n=2$ and $0 \leq p \leq 1$

\begin{tabular}{c|ccc|ccc|}
\multicolumn{4}{c}{$\operatorname{RwS}$} \\
Payoff $\boldsymbol{V}$ & $\mathbf{1 2}$ & $\mathbf{8}$ & $\mathbf{0}$ & $\mathbf{1 6}$ & $\mathbf{8}$ & $\mathbf{4}$ \\
Prob. $P(V)$ & $p$ & $(1-p) p$ & $(1-p)^{2}$ & $p^{2}$ & $(1-p) p$ & $(1-p)$ \\
\cline { 2 - 7 } & & &
\end{tabular}

\begin{tabular}{|c|c|c|c|c|c|c|}
\hline \multirow[b]{2}{*}{ Payoff $V$} & \multicolumn{2}{|c|}{ RS, SR } & $\mathrm{SS}$ & \multicolumn{3}{|c|}{$\mathrm{RR}$} \\
\hline & 12 & 4 & 8 & 16 & 8 & $\mathbf{0}$ \\
\hline Prob. $P(V)$ & $p$ & $(1-p)$ & 1 & $p^{2}$ & $2(1-p) p$ & $(1-p)^{2}$ \\
\hline
\end{tabular}

Figure 1 shows how the switching rate between RwS and the other strategies varies with $\alpha$. We see that RwS has an advantage over all other strategies for $0<\alpha<1$, suggesting that RwS should be able to win any evolutionary competition in these environments. Again, this prediction is supported by the simulation results in Section 5.

\subsection{Arbitrary $p$}

We now consider the case where $p$, the probability of winning the risky lottery, is any number between 0 and 1 (while the risky lottery's expected value is between 0 and 8). Table IV gives the probability distributions for each pure strategy.

We can also construct a new probability matrix for each pairing such as the one for RwS vs. RR shown in Table V. We can then compute the switching rate for our pairing as before. For example, the switching rate for RwS vs. RR is now the following.

$$
\begin{aligned}
\nabla_{\alpha}(\mathrm{RwS}, \mathrm{RR})= & |0-0|^{\alpha} \operatorname{sign}(0-0) \cdot(1-p)^{4} \\
& +|8-0|^{\alpha} \operatorname{sign}(8-0) \cdot(1-p)^{3} p \\
& +|12-0|^{\alpha} \operatorname{sign}(12-0) \cdot(1-p)^{2} p
\end{aligned}
$$


Table V.

Payoff Distribution for RwS vs. RR in the Sequential Lottery Game with $n=2$ and Arbitrary $p$. Entries in the Table Show Give the Probabilities That Each Pair of Payoffs Occurs

$\mathrm{RR}$

\begin{tabular}{|c|c|c|c|}
\cline { 2 - 4 } \multicolumn{1}{c|}{} & $\mathbf{1 2}$ & $\mathbf{8}$ & $\mathbf{0}$ \\
\hline $\mathbf{1 6}$ & $p^{3}$ & $(1-p) p^{3}$ & $(1-p)^{2} p^{2}$ \\
\hline $\mathbf{8}$ & $2(1-p) p^{2}$ & $2(1-p)^{2} p^{2}$ & $2(1-p)^{3} p$ \\
\hline $\mathbf{0}$ & $(1-p)^{2} p$ & $(1-p)^{3} p$ & $(1-p)^{4}$ \\
\hline
\end{tabular}

$$
\begin{aligned}
& +|0-8|^{\alpha} \operatorname{sign}(0-8) \cdot 2(1-p)^{3} p \\
& +|8-8|^{\alpha} \operatorname{sign}(8-8) \cdot 2(1-p)^{2} p^{2} \\
& +|12-8|^{\alpha} \operatorname{sign}(12-8) \cdot 2(1-p) p^{2} \\
& +|0-16|^{\alpha} \operatorname{sign}(0-16) \cdot(1-p)^{2} p^{2} \\
& +|8-16|^{\alpha} \operatorname{sign}(8-16) \cdot(1-p) p^{3} \\
& +|12-16|^{\alpha} \operatorname{sign}(12-16) \cdot p^{3} \\
& =4^{\alpha}\left(2(1-p) p^{2}-p^{3}\right)+8^{\alpha}\left((1-p)^{3} p-2(1-p)^{3} p-(1-p) p^{3}\right) \\
& +12^{\alpha}(1-p)^{2} p+16^{\alpha}(1-p)^{2} p^{2} .
\end{aligned}
$$

Notice that when $p>0.5$, the risky lottery has a higher expected value than the safe lottery, and the opposite is true when $p<0.5$. Thus, RR has the highest expected value when $p>0.5$, and SS has the highest expected value when $p<0.5$.

Surprisingly, even though $\mathrm{RwS}$ has a suboptimal expected value when $p \neq 0.5$, by examining the switching rates, we can see that it still has an evolutionary advantage over both SS and RR for many values of $p$ and $\alpha$. Figure 2 shows the values of $p$ and $\alpha$ for which $\nabla_{\alpha}(\mathrm{RwS}, \mathrm{RR})>0$ and $\nabla_{\alpha}(\mathrm{RwS}, \mathrm{SS})>0$, meaning that for these values of $p$ and $\alpha$, RwS has an evolutionary advantage over expected-value maximizing strategies. Under the imitate-the-better dynamic $(\alpha=0)$, the range is surprisingly large. For example, RwS outperforms SS even when $p=0.4$, and SS has a significantly higher expected value than RwS. As $\alpha$ increases, the range shrinks at a roughly linear rate, disappearing at $\alpha=1$ (i.e., the replicator dynamic).

\subsection{Evolutionary Stability of State-Dependent Risk Behavior}

In this section, we discuss whether RwS is an evolutionarily stable strategy (ESS) in the 2-lottery game, when $p=0.5$ and $0<\alpha<1$. We first give the classical definition of an ESS, explain why it cannot be directly applied to $n$ lottery games when $\alpha \neq 1$, and propose an intuitive modification to make it applicable. We then show that RWS fits our modified definition of an ESS.

4.5.1 Evolutionary Stability under Imitation Dynamics. In an evolutionary game, a population of agents using an evolutionarily stable strategy (ESS) is resilient against a small invading population using any other strategy [Smith 1982]. According to Maynard Smith, strategy $S$ is an ESS if for every strategy 


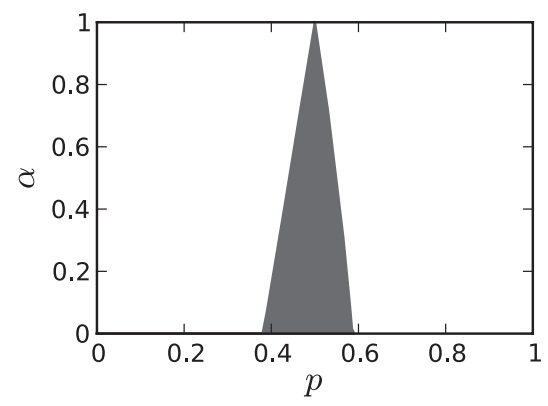

Fig. 2. The shaded area indicates values of $p$ and $\alpha$ for which the switching rate between RwS and the expected-value maximizing strategy (i.e., RR if $p \geq 0.5$, SS otherwise) is greater than 0 . $\mathrm{RWS}$ is at a disadvantage in terms of expected utility when $p \neq 0.5$, but it still manages to retain its evolutionary advantage for a wide range of values.

$T \neq S$, one of the following conditions holds:

$-E(S, S)>E(T, S)$,

$-E(S, S)=E(T, S)$ and $E(S, T)>E(T, T)$,

where $E(X, Y)$ is the expected payoff an agent receives by playing strategy $X$ against strategy $Y$ [Smith 1982].

This definition does not apply directly to the $n$-lottery game because it assumes we are using the replicator dynamic for which the expected payoff of a strategy pairing is all that is necessary to determine whether one strategy will perform better than the other. As shown in Section 4.4, with population dynamics other than the replicator dynamic, the expected value of a pairing is not sufficient to determine which strategy will perform better in the $n$-lottery game. Thus, we will use the switching rate $\nabla_{\alpha}(X, Y)$ rather than $E(X, Y)$ when defining evolutionary stability for the $n$-lottery game under imitation dynamics. We believe this is appropriate because if there are many agents using $X$ and few using $Y$, then

$-\nabla_{\alpha}(X, X)>\nabla_{\alpha}(Y, X)$ implies that $X$ will grow faster playing against itself than $Y$ will grow playing against $X$, so $Y$ will not be able to gain population and will eventually die off;

$-\nabla_{\alpha}(X, X)=\nabla_{\alpha}(Y, X)$ and $\nabla_{\alpha}(X, Y)>\nabla_{\alpha}(Y, Y)$ implies that $X$ and $Y$ grow at the same rate when playing against $X$, but $X$ grows faster than $Y$ when playing against $Y$, so $Y$ will still not be able to gain population and will eventually die off.

These scenarios correspond to the two conditions for $X$ to be an ESS in the classical definition.

Replacing expected value with switching rates in the preceding definition gives us the following two conditions for $S$ to be an ESS:

$-\nabla_{\alpha}(S, S)>\nabla_{\alpha}(T, S)$ or

$-\nabla_{\alpha}(S, S)=\nabla_{\alpha}(T, S)$ and $\nabla_{\alpha}(S, T)>\nabla_{\alpha}(T, T)$

ACM Transactions on Intelligent Systems and Technology, Vol. 1, No. 1, Article 6, Publication date: October 2010. 


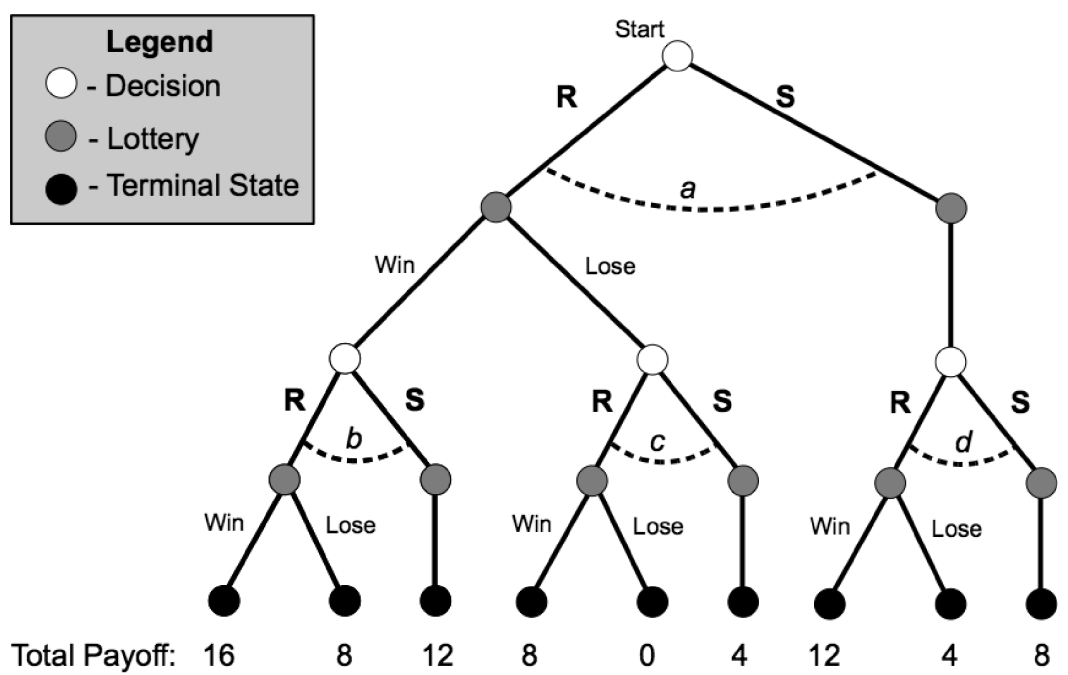

Fig. 3. Representation of the 2-lottery game as a game tree consisting of decision nodes in which the agent chooses between a risky $(\mathbf{R})$ and safe $(\mathbf{S})$ lottery, lottery nodes, and terminal nodes, which assign the agent its total payoff. Any strategy in this game can be represented as $S_{a, b, c, d}$, where $a, b, c$, and $d$ give the probabilities of choosing the risky lottery at each of the four corresponding decision nodes.

for all $T \neq S$. Since $\nabla_{\alpha}(S, S)=0$ and $\nabla_{\alpha}(S, T)=-\nabla_{\alpha}(T, S)$ for all $S$ and $T$, we can combine these conditions and define ESS in the $n$-lottery game as follows.

Definition 1. A strategy $S$ is an evolutionarily stable strategy (ESS) in the $n$-lottery game if, for any strategy $T \neq S, \nabla_{\alpha}(S, T)>0$.

4.5.2 RWS is Evolutionarily Stable. In this section, we show that RwS is an ESS by Definition 1 for the 2-lottery game with $p=0.5$ and $0<\alpha<1$. To do this, we must show that it has a positive switching rate with an arbitrary strategy. Therefore, our first step must be to devise a method for representing an arbitrary strategy for the 2-lottery game. We have found that, if the decisions an agent makes and the possible lottery outcomes are arranged into a game tree as shown in Figure 3, then any strategy can be expressed as $S_{a, b, c, d}$, where $a, b, c$, and $d$ give the agent's probability of choosing the risky lottery at each of the four decision nodes indicated in the figure. For instance, the pure strategies we have been dealing with thus far can be represented as follows (here, a "-" in place of one of the four probabilities indicates that any value is acceptable since the decision corresponding to that probability is never reached).

- $\mathrm{SS}$ is $S_{0,-,-, 0}$

$-\mathrm{RR}$ is $S_{1,1,1,-}$

- RS is $S_{1,0,0,-}$

-SR is $S_{0,-,-1}$

- RwS is $S_{1,0,1,-}$

- $\mathrm{RwR}$ is $S_{1,1,0,-}$

ACM Transactions on Intelligent Systems and Technology, Vol. 1, No. 1, Article 6, Publication date: October 2010. 
We can now calculate the switching rate between RwS and an arbitrary strategy $S_{a, b, c, d}$ in terms of $a, b, c, d$, and $\alpha$ in the same way we calculated $\nabla_{\alpha}(\mathrm{RwR}, \mathrm{RR})$ and $\nabla_{\alpha}(\mathrm{RwR}, \mathrm{SS})$ in Section 4.3. This comes out to: ${ }^{6}$

$$
\begin{aligned}
\nabla_{\alpha}\left(\operatorname{RwR}, S_{a, b, c, d}\right)= & {\left[\frac{1}{8}\left(2 * 8^{\alpha}-12^{\alpha}-4^{\alpha}\right)\right](a(1-c)+(1-a) d) } \\
& +\left[\frac{1}{4}\left(2 * 4^{\alpha}-8^{\alpha}\right)\right](1-a)(1-d) \\
& +\left[\frac{1}{16}\left(2 * 12^{\alpha}+2 * 4^{\alpha}-2 * 8^{\alpha}-16^{\alpha}\right)\right] a b .
\end{aligned}
$$

The three bracketed terms are all strictly greater than 0 for $0<\alpha<1$; in fact, they are identical to the curves in Figure 1 for SR/RS, SS, and RR, respectively. Intuitively, this says that the switching rate for the arbitrary strategy is just the probability that the strategy follows the pure strategies SS, SR, RS, and RR, times the switching rate between RwS and each of those strategies. Given that the bracketed terms are strictly positive, it is easy to see that $\nabla_{\alpha}\left(\mathrm{RwR}, S_{a, b, c, d}\right)>$ 0 unless $a=1, b=0$, and $c=1$. Since $S_{1,0,1,-}$ is equivalent to $\mathrm{RWS}$, this means that $\nabla_{\alpha}\left(\mathrm{RwR}, S_{a, b, c, d}\right)>0$ for all $S_{a, b, c, d} \neq \mathrm{RwS}$. Thus, RwS is an evolutionarily stable strategy by Definition 1.

\subsection{Other Values of $n$}

With the exception of Section 4.5.1, our theoretical development has been largely restricted to the case $n=2$. We now discuss briefly what happens for other values of $n$.

The case $n=1$ is relatively trivial: there are only two pure strategies, both are unconditional, and both perform equally well (for more details, see Roos and Nau [2010a]).

The case $n>2$ is very hard to analyze because the number of pure strategies is super-exponential in $n$. However, intuition suggests that the behavior pattern exhibited by $\mathrm{RwS}$ for $n=2$, namely, to play it safe when having done well and risky otherwise, should also have an advantage when $n>2$. We discuss some pilot experiments that support this in Section 5.3.

\section{SIMULATIONS}

To further investigate the population dynamics in a population consisting of all pure strategies, we ran computer simulations of agent-based models playing our two-choice evolutionary lottery game. These simulations and results are described in the next section.

\subsection{Setup and Implementation}

Our simulations for the two-choice lottery game environment explore population evolution under a variety of parameter combinations of $\alpha$ (the imitation parameter) and $p$ (the probability of winning the risky lottery). The types of

${ }^{6}$ We give a full derivation in the appendix. 


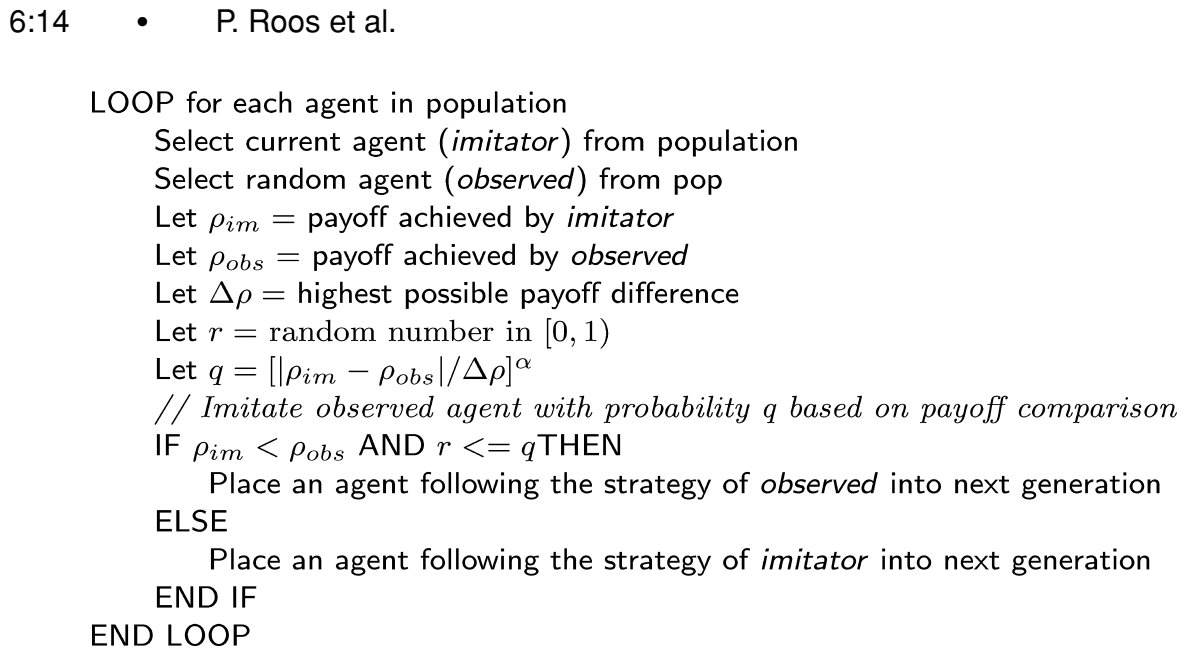

Fig. 4. Pseudocode for the pairwise comparison imitation process used to reproduce agents into the next generation in our evolutionary simulations. Takes parameter $0 \leq \alpha \leq 1$.

agents included were the six pure strategies for the two-choice game described earlier. All simulations started with an initial population of 1000 agents for each agent type.

To model the imitation dynamics given by Eq. (2) in our finite population agent-based model, we used a pairwise comparison process [Traulsen and Hauert 2009] to model the transmission of strategies among agents. In each generation, after all agents have received payoffs from chosen lotteries, each agent $i$ compares its (individual) payoff $\rho(i)$ to that of a randomly drawn agent from the population $j$ (with payoff $\rho(j)$ ) and adopts the strategy of this agent with a probability $q$ if $\rho(j)>\rho(i)$. In order to achieve the parameterized dynamics given by Eq. (1) in our agent-based model, we use

$$
q=[|\rho(j)-\rho(i)| / \Delta \rho]^{\alpha}, \quad 0 \leq \alpha \leq 1,
$$

where $\Delta \rho$ is the highest possible difference in payoff. Figure 4 provides pseudocode on how this pairwise comparison imitation process was implemented.

\subsection{Results}

Figure 5(a,b,c) shows the results for simulations with $p=0.5$ for each $\alpha=1$ (the replicator dynamic), $\alpha=0$ (the imitate-the-better dynamic), and $\alpha=0.5$. Each plot is an average over 20 simulation runs (the amount of variation from one run to another was quite small). These experiments confirm our analysis from Section 4.3, which shows that RwS has an evolutionarily advantageous risk behavior under any $0 \leq \alpha<0$.

As predicted by our analysis, RwS outperformed the other strategies evolutionarily except when $\alpha=1$.

-For $\alpha=1$, all of the strategies performed equally well and remained at their initial population counts.

- For $\alpha=0$, the state-dependent strategy RwS outperformed the other strategies. $\mathrm{RwS}$ rose in population proportion relatively quickly to comprise the 


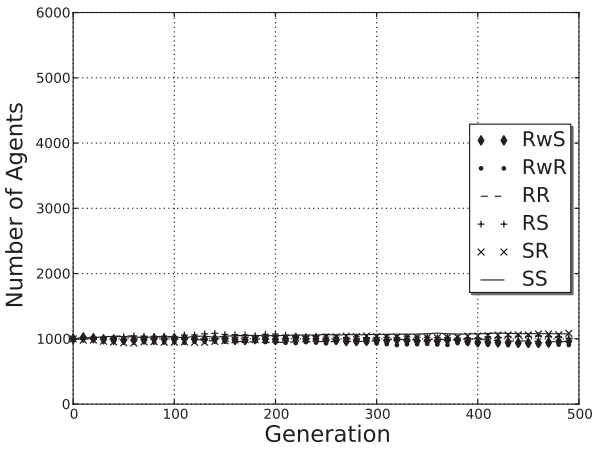

a) $\alpha=1$

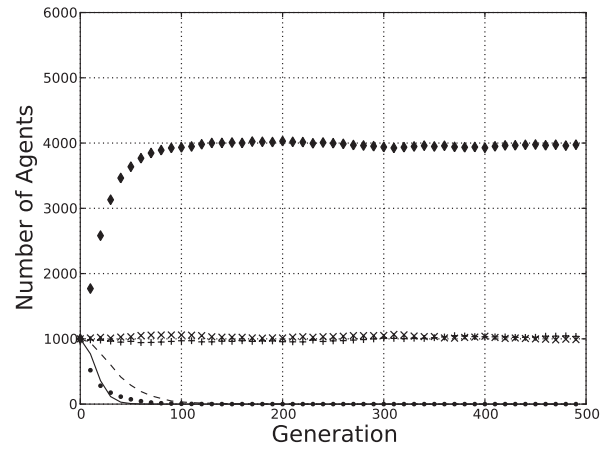

b) $\alpha=0$

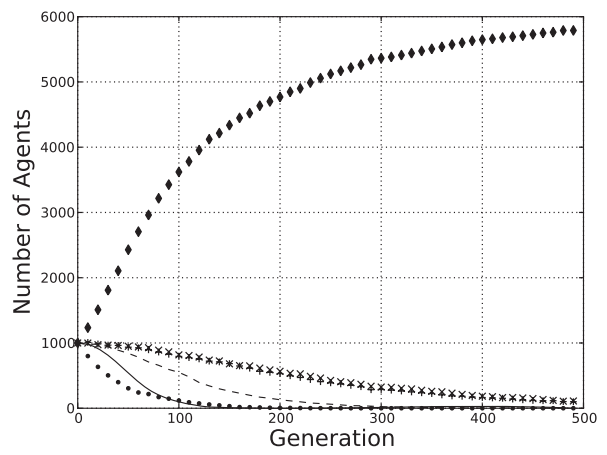

c) $\alpha=0 \triangleright 5$

Fig. 5. Results of simulations $1 \alpha$ values for the population dynamic and $p=0.5$. Each plot shows the number of agents of each type over the course of evolution for 500 generations. Simulation runs for $\alpha=0.2,0.4,0.6$, and 0.8 produced qualitatively identical results to the case of $\alpha=0.5$.

majority $(>2 / 3)$ of the population and remained there throughout subsequent generations. Furthermore, the two unconditional strategies SR and RS remained, comprising the proportion of the population not taken over by $\mathrm{RwS}$.

- For $\alpha=0.5$, the RwS agent population grew similarly as for $\alpha=0$, but here RwS also had an advantage against SR and RS (as indicated by Figure 1) and thus continued to grow to comprise $100 \%$ of the population.

We also ran simulations with $\alpha=0.2,0.4,0.6$, and 0.8 . The results for these $\alpha$ values are all essentially equal to the case of $\alpha=0.5$. The only difference is that the rate at which RwS grows to take over the population is inversely related to $\alpha$ (i.e., for larger $\alpha$ values, it takes longer for $\mathrm{RWS}$ to take over the population).

In order to explore lottery games in which the risky lottery has a different expected value than the safe lottery, we also ran experiments with $p=0.3$, $0.4,0.55$, and 0.7. These values were chosen because for $\alpha=0$, two of them lie within $(p=0.4,0.55)$, the shaded area of Figure 2 , and the other two 


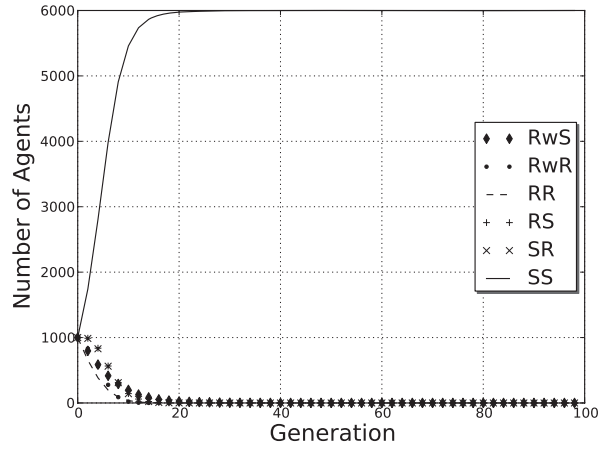

a) $p=0 \triangleright 3$

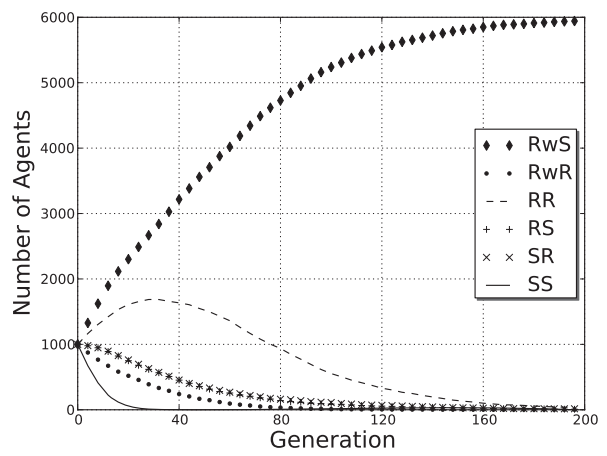

c) $p=0 \triangleright 55$

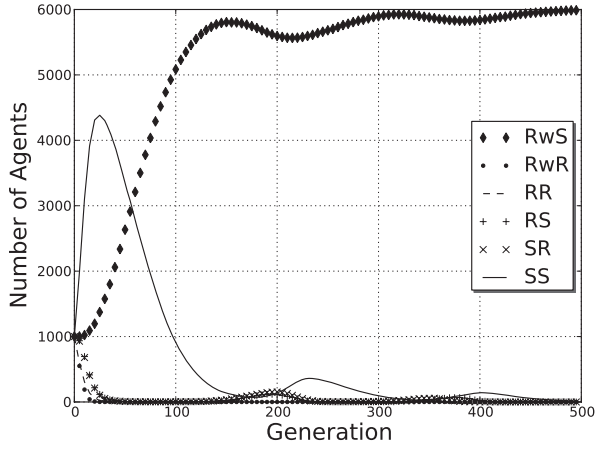

b) $p=0 \triangleright 4$

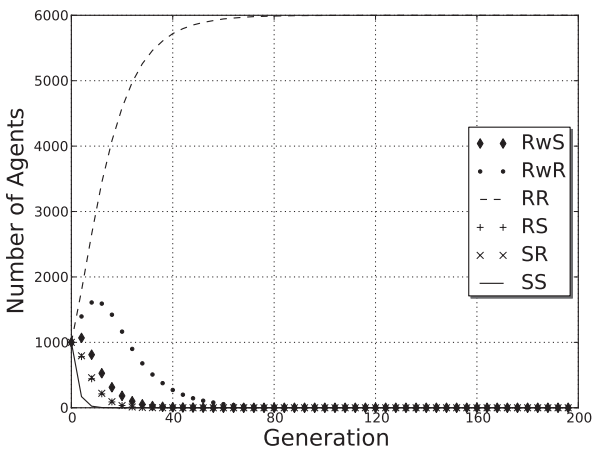

d) $p=0 \triangleright 7$

Fig. 6. Results of simulations with four different $p$ values for the risky lottery and $\alpha=0$. Each plot shows the number of agents of each type over the course of evolution for 500 generations.

( $p=0.3,0.7$ ) lie outside this area. Recall that the shaded area is the region for which our analysis predicts that RwS has an evolutionary advantage over the expected-value-maximizing strategy, and the unshaded areas are regions for which our analysis predicts the reverse.

As shown in Figure 6(a,b,c,d), the simulation results confirm the theoretical predictions. More specifically:

—for $p=0.2$ (Figure 6(a)), SS is the expected-value maximizing strategy and it takes over the population;

- for $p=0.7$ (Figure $6(\mathrm{~d})$ ), RR is the expected-value maximizing strategy and it takes over the population;

- even though SS is the expected-value-maximizing strategy for $p=0.4$ (Figure 6(b)) and RR is for $p=0.55$ (Figure 6(c)), in both cases RwS has an evolutionary advantage and takes over the population.

In Figures 6(b,c,d), some fluctuations occur before stabilization. These occur because of the differing amounts of evolutionary advantage that different strategies have over others. For example, a strategy $a$ may grow in number temporarily because it has an advantage over another strategy $b$. But once $b$ becomes 
Table VI. Pure Strategies Included in Our Lottery Game Simulations for

$$
n=4
$$

\begin{tabular}{l|l}
\hline Strategy & \multicolumn{1}{c}{ Behavior } \\
\hline SSSS & always choose safe \\
RRRR & always choose risky \\
SSRR & choose safe in lotteries 1 \& 2, then choose risky in 3 \& 4 \\
RRSS & choose risky in lotteries 1 \& 2, then choose safe in $3 \& 4$ \\
RRwSS & $\begin{array}{l}\text { choose risky in lotteries } 1 \& 2, \\
\text { then choose safe in 3 \& 4 only if } 1 \& 2 \text { were won, else choose risky }\end{array}$ \\
RRwRR & $\begin{array}{l}\text { choose risky in lotteries 1 \& 2, } \\
\text { then choose risky in 3 \& 4 only if } 1 \& 2 \text { were lost, else choose safe }\end{array}$ \\
\hline
\end{tabular}

extinct (or sufficiently small in number), $a$ will diminish because some other strategy $c$ has an advantage over $a$.

\subsection{Simulations for $n>2$}

In Section 4.6, we hypothesized that RwS's behavior pattern, namely, to be riskaverse when it has done well and risk-seeking when it has done badly, may be advantageous in lottery games with $n>2$. To test this hypothesis, we ran a pilot experiment for the case $n=4$, using the six strategies shown in Table VI.

For our simulations, we used an initial population of 1000 agents of each type, and the parameters $p=0.5$ for the risky lottery and $\alpha=0$ for the population dynamic. The results were qualitatively the same as the ones in Figure 5(c): the RRwSS strategy dominated the other strategies and grew to comprise $100 \%$ of the population. This would seem to confirm our hypothesis, but since there are hundreds of pure strategies when $n=4$ and we only looked at six of the simpler ones, the result should be regarded as purely preliminary.

\section{CONCLUSIONS}

Our results show that for imitation dynamics other than the pure replicator dynamic, there are evolutionary game environments in which the RwS strategy has an evolutionary advantage over expected-value maximization. Since RwS's risk-taking behavior is similar to the risk preferences captured in several prominent models of human decision making, this suggests that population dynamics other than the replicator dynamic may model an important mechanism for the emergence of those risk preferences.

There are several ways in which our work could be extended.

- Our study was restricted to simple lottery games in order to avoid confounding factors that may be present in more complex social games (e.g., trust, reputation, kinship, etc.). It would be interesting to explore the relationships among reproduction dynamics, risk preferences, and social interactions such as cooperation. We have already begun some work along this line: Roos and Nau [2010a] shows that risk behavior similar to RwS can promote the evolution of cooperation in a situation where the cooperation requires a risky decision (namely, choosing to cooperate).

- It will be important to examine other population dynamics in which a strategy's reproductive success is not always proportional to its expected payoff. 
For example, if a death rate (e.g., Nowak and Sigmund [1993]) is implemented as a payoff-dependent threshold function, we might expect risk propensities to differ depending on whether an agent is above or below that threshold in a manner similar to behavior above or below an aspiration level in SP/A theory.

- Our study focused on the case $n=2$, that is, in each generation the agents made two decisions. We believe that state-dependent risk preferences like those of RwS should also have an advantage when $n>2$, and Section 5.3 discussed some pilot experiments that support this intuition. Conducting more extensive studies will be a topic for future work.

-We assumed a well-mixed population in which every agent was able and equally likely to imitate any other. It would be interesting to explore the possible effects of social or physical structures (that may guide or constrain imitation) on the evolution of risk behavior.

-Finally, it may be useful to conduct empirical studies of which type of imitation dynamics best models human imitation propensities. The insights of this article combined with such knowledge have potential application in domains where human decision making under risk is of interest, including economics, medicine, military and public policy.

\section{APPENDIX}

In this section, we present the derivation of $\nabla_{\alpha}\left(\mathrm{RwS}, S_{a, b, c, d}\right)$ used in Section 4.5. Using Table IV and Figure 3, we can determine the probability of each pair of payoffs occurring and use them for the values of $p(r, s)$ as follows.

$$
\begin{aligned}
& \nabla_{\alpha}\left(\mathrm{RWS}, S_{a, b, c, d}\right)=|12-8|^{\alpha}(p)(a p b(1-p)+a p c(1-p)+(1-a)(1-d)) \\
&+|12-4|^{\alpha}(p)(a(1-p)(1-c)+(1-a) d(1-p)) \\
&+|12-0|^{\alpha}(p)(a(1-p) c(1-p)) \\
&+|8-4|^{\alpha}(p(1-p))(a(1-p)(1-c)+(1-a) d(1-p)) \\
&+|8-0|^{\alpha}(p(1-p))(a(1-p) c(1-p)) \\
&-|16-12|^{\alpha} p(a p b p) \\
&-|16-8|^{\alpha}(1-p) p(a p b p) \\
&-|16-0|^{\alpha}(1-p)^{2}(a p b p) \\
&-|12-8|^{\alpha}(1-p) p(a p(1-b)+(1-a) d p) \\
&-|12-0|^{\alpha}(1-p)^{2}(a p(1-b)+(1-a) d p) \\
&-|8-0|^{\alpha}(1-p)^{2}(a p b(1-p)+a(1-p) c p+(1-a)(1-d)) \\
&-|4-0|^{\alpha}(1-p)^{2}(a(1-p)(1-c)+(1-a) d(1-p))
\end{aligned}
$$

Since we are considering the case where $p=0.5$, we can collect terms as though $p=(1-p)$ (for ease of exposition we will wait to substitute 0.5 for $p)$ :

$$
\begin{aligned}
\nabla_{\alpha}\left(\mathrm{RwS}, S_{a, b, c, d}\right)= & |16|^{\alpha}\left(0-a b p^{4}\right)+|12|^{\alpha}\left(a c p^{3}-a(1-b) p^{3}-(1-a) d p^{3}\right) \\
& +\left(|4|^{\alpha} p-|8|^{\alpha} p^{2}\right)\left(a b p^{2}+a c p^{2}+(1-a)(1-d)\right) \\
& +\left(|8|^{\alpha} p+|4|^{\alpha} p^{2}-|4|^{\alpha} p^{2}\right)(a(1-c) p+(1-a) d p)
\end{aligned}
$$




$$
\begin{aligned}
& +|8|^{\alpha}\left(a c p^{4}+a b p^{4}\right) \\
& +|4|^{\alpha}\left(0-a b p^{3}-a(1-b) p^{3}-(1-a) d p^{3}\right),
\end{aligned}
$$

which yields

$$
\begin{aligned}
\nabla_{\alpha}\left(\mathrm{RwS}, S_{a, b, c, d}\right)= & -|16|^{\alpha}\left(a b p^{4}\right)+|12|^{\alpha}\left(a c p^{3}-a(1-b) p^{3}-(1-a) d p^{3}\right) \\
+ & |8|^{\alpha}\left(a c p^{4}+a(1-c) p^{2}+(1-a) d p^{2}\right. \\
& \left.-a b p^{4}-a b p^{4}-a c p^{4}-(1-a)(1-d) p^{2}\right) \\
& +|4|^{\alpha}\left(a b p^{3}+a c p^{3}+(1-a)(1-d) p-a b p^{3}-a(1-b) p^{3}\right. \\
& \left.-(1-a) d p^{3}\right),
\end{aligned}
$$

which yields

$$
\begin{aligned}
\nabla_{\alpha}\left(\mathrm{RWS}, S_{a, b, c, d}\right)= & -|16|^{\alpha}\left(a b p^{4}\right)+|12|^{\alpha}\left(a c p^{3}-a(1-b) p^{3}-(1-a) d p^{3}\right) \\
& +|8|^{\alpha}\left(a(1-c) p^{2}+(1-a) d p^{2}-a b p^{4}-a b p^{4}-(1-a)(1-d) p^{2}\right) \\
& +|4|^{\alpha}\left(a c p^{3}+(1-a)(1-d) p-a(1-b) p^{3}-(1-a) d p^{3}\right) .
\end{aligned}
$$

Recollecting terms gives us

$$
\begin{aligned}
\nabla_{\alpha}\left(\mathrm{RwS}, S_{a, b, c, d}\right)= & \left(12^{\alpha}+4^{\alpha}\right) a c p^{3}+8^{\alpha} a(1-c) p^{2} \\
& +4^{\alpha}(1-a)(1-d) p+8^{\alpha}(1-a) d p^{2} \\
& -\left(4^{\alpha}+12^{\alpha}\right)(1-a) d p^{3}-8^{\alpha}(1-a)(1-d) p^{2} \\
& -\left(16^{\alpha}+2 * 8^{\alpha}\right) a b p^{4}-\left(12^{\alpha}+4^{\alpha}\right) a(1-b) p^{3} .
\end{aligned}
$$

Substituting $p=\frac{1}{2}$ and expanding the final term, we get

$$
\begin{aligned}
\nabla_{\alpha}\left(\mathrm{RWS}, S_{a, b, c, d}\right)= & \frac{1}{8}\left(12^{\alpha}+4^{\alpha}\right) a c+\frac{1}{4} 8^{\alpha} a(1-c) \\
& +\frac{1}{4}\left(2 * 4^{\alpha}-8^{\alpha}\right)(1-a)(1-d)+\frac{1}{8}\left(2 * 8^{\alpha}-4^{\alpha}-12^{\alpha}\right)(1-a) d \\
& -\frac{1}{16}\left(16^{\alpha}+2 * 8^{\alpha}\right) a b-\frac{1}{8}\left(12^{\alpha}+4^{\alpha}\right) a+\frac{1}{8}\left(12^{\alpha}+4^{\alpha}\right) a b,
\end{aligned}
$$

which yields

$$
\begin{aligned}
\nabla_{\alpha}\left(\operatorname{RwS}, S_{a, b, c, d}\right)= & \frac{1}{8}\left(12^{\alpha}+4^{\alpha}\right)(a c-a)+\frac{1}{4} 8^{\alpha} a(1-c) \\
& +\frac{1}{4}\left(2 * 4^{\alpha}-8^{\alpha}\right)(1-a)(1-d)+\frac{1}{8}\left(2 * 8^{\alpha}-4^{\alpha}-12^{\alpha}\right)(1-a) d \\
& +\frac{1}{16}\left(2 * 12^{\alpha}+2 * 4^{\alpha}-2 * 8^{\alpha}-16^{\alpha}\right) a b .
\end{aligned}
$$

Since $(a c-a)=-a(1-c)$, this yields

$$
\begin{aligned}
\nabla_{\alpha}\left(\mathrm{RwS}, S_{a, b, c, d}\right)= & \frac{1}{8}\left(2 * 8^{\alpha}-4^{\alpha}-12^{\alpha}\right) a(1-c) \\
& +\frac{1}{4}\left(2 * 4^{\alpha}-8^{\alpha}\right)(1-a)(1-d)+\frac{1}{8}\left(2 * 8^{\alpha}-4^{\alpha}-12^{\alpha}\right)(1-a) d \\
& +\frac{1}{16}\left(2 * 12^{\alpha}+2 * 4^{\alpha}-2 * 8^{\alpha}-16^{\alpha}\right) a b .
\end{aligned}
$$

ACM Transactions on Intelligent Systems and Technology, Vol. 1, No. 1, Article 6, Publication date: October 2010. 
Finally, recollecting terms gives us

$$
\begin{aligned}
\nabla_{\alpha}\left(\operatorname{RwS}, S_{a, b, c, d}\right)= & \frac{1}{8}\left(2 * 8^{\alpha}-12^{\alpha}-4^{\alpha}\right)(a(1-c)+(1-a) d) \\
& +\frac{1}{4}\left(2 * 4^{\alpha}-8^{\alpha}\right)(1-a)(1-d) \\
& +\frac{1}{16}\left(2 * 12^{\alpha}+2 * 4^{\alpha}-2 * 8^{\alpha}-16^{\alpha}\right) a b,
\end{aligned}
$$

which matches Eq. (3).

\section{ACKNOWLEDGMENTS}

We wish to thank the anonymous reviewers for their helpful suggestions.

\section{REFERENCES}

AвEL, A. 1990. Asset prices under habit formation and catching up with the Joneses. Amer. Econ. Rev. 80, 2, 38-42.

AleXANDER, J. M. 2009. Evolutionary game theory. In The Stanford Encyclopedia of Philosophy, Fall 2009 Ed., E. N. Zalta, Ed.

Apesteguia, J., Huck, S., AND Oechssler, J. 2007. Imitation-theory and experimental evidence. J. Econ. Theory 136, 1, 217-235.

ARrow, K. J. 1971. Essays in the Theory of Risk-Bearing. Markham, Chicago.

Axelrod, R. and Hamilton, W. D. 1981 . The evolution of cooperation. Science 211, 4489, 13901396.

Binmore, K. 1998. The evolution of fairness norms. Rationality and Society 10, 275-302.

Blume, L. 1993. The statistical mechanics of strategic interaction. Games Econ. Behav. 5, 3, 387-424.

Bowles, S. ANd GiNTis, H. 2004. The evolution of strong reciprocity: Cooperation in heterogeneous populations. Theoret. Population Biol. 65, 1, 17-28.

DAwID, H. 2007. Evolutionary game dynamics and the analysis of agent-based imitation models: The long run, the medium run and the importance of global analysis. J. Econ. Dynam. Control 31, 6, 2108-2133.

Eguluz, V. M., Zimmermann, M. G., Cela-Conde, C. J., and Miguel, M. S. 2005 . Cooperation and emergence of role differentiation in the dynamics of social networks. Amer. J. Sociol. 110, 977.

Epstein, L. AND ZiN, S. 1989. Substitution, risk aversion, and the temporal behavior of consumption and asset returns: A theoretical framework. Econometrica: J. Econometric Soc., 937-969.

Epstein, L. ANd Zin, S. 1991. Substitution, risk aversion, and the temporal behavior of consumption and asset returns: An empirical analysis. J. Political Econ., 263-286.

Fang, C., Kimbrough, S. O., Pace, S., Valluri, A., and Zheng, Z. 2002. On adaptive emergence of trust behavior in the game of stag hunt. Group Decision Negotiation 11, 6, 449-467.

Friedman, M. And Savage, L. J. 1948. The utility analysis of choices involving risk. J. Political Econ. 56, 4, 279-304.

Gintis, H. 2000. Game Theory Evolving. Princeton University Press, Princeton, New Jersey.

Hauert, C. and Szabo, G. 2005. Game theory and physics. Amer. J. Physics 73, 5, 405-414.

Hofbauer, J. And Sigmund, K. 1998. Evolutionary Games and Population Dynamics. Cambridge University Press, Cambridge, UK.

Huberman, B. A. and Glance, N. S. 1993. Evolutionary games and computer simulations. Proc. Nat. Acad. Sciences USA 90, 16, 7716-7718.

Huck, S., Normann, H., AND Oechssler, J. 2000. Does information about competitors' actions increase or decrease competition in experimental oligopoly markets? Int. J. Indust. Organ. 18, 1, 39-57.

Huck, S., Normann, H. T., ANd Oechssler, J. 1997. Learning in cournot oligopoly: An experiment. SSRN eLibrary.

ACM Transactions on Intelligent Systems and Technology, Vol. 1, No. 1, Article 6, Publication date: October 2010. 
Kahneman, D. And Tversky, A. 1979. Prospect theory: An analysis of decision under risk. Econometrica 47, 263-291.

KImbrough, S. O. 2005. Foraging for trust: Exploring rationality and the stag hunt game. In Trust Management. 1-16.

Loomes, G. And Sugden, R. 1982. Regret theory: An alternative theory of rational choice under uncertainty. Economic J. 92, 805-824.

LoPES, L. L. 1987. Between hope and fear: The psychology of risk. In Advances in Experimental Social Psychology, vol. 20, 255-295.

LOPES, L. L. 1990. Re-modeling risk aversion. In Acting Under Uncertainty: Multidisciplinary Conceptions, G. M. von Furstenberg, Ed., Kluwer, Boston, 267-299.

Lopes, L. L. And Oden, G. C. 1999. The role of aspiration level in risky choice: A comparison of cumulative prospect theory and sp/a theory. J. Math. Psychol. 43, 286-313.

Nowak, M. A., Bonhoeffer, S., ANd May, R. M. 1994. Spatial games and the maintenance of cooperation. Proc. Nat. Acad. Sciences USA 91, 11, 4877-4881.

NowaK, M. A. AND May, R. M. 1992. Evolutionary games and spatial chaos. Nature 359, 6398, 826-829.

NowAK, M. A. AND Sigmund, K. 1993. A strategy of win-stay, lose-shift that outperforms tit for tat in the prisoner's dilemma game. Nature 364, 56-58.

Offerman, T., Potters, J., AND Sonnemans, J. 2002. Imitation and belief learning in an oligopoly experiment. Rev. Econ. Studies 69, 4, 973-997.

PaGe, K. And NowaK, M. 2002. Empathy leads to fairness. Bull. Math. Biol. 64, 6, 1101-1116.

RABIN, M. 2000. Risk aversion and Expected-Utility theory: A calibration theorem. Econometrica $68,5,1281-1292$.

Riolo, R. L., Cohen, M. D., AND AxELRod, R. 2001. Evolution of cooperation without reciprocity. Nature 411, 441-443.

Roos, P. AND NAU, D. S. 2009. Conditionally risky behavior vs expected value maximization in evolutionary games. In Proceedings of the 6th Conference of the European Social Simulation Association (ESSA '09).

Roos, P. AND NAU, D. S. 2010a. Risk preference and sequential choice in evolutionary games. Advances Complex Syst. To appear.

Roos, P. AND NAU, D. S. 2010b. State-dependent risk preferences in evolutionary games. In Proceedings of the International Conference on Social Computing, Behavioral Modeling, and Prediction.

SchlaG, K. 1999. Which one should I imitate? J. Math. Econ. 31, 4, 493-522.

ScHLAG, K. H. 1998. Why imitate, and if so, how? a bounded rational approach to Multi-Armed bandits. J. Econ. Theory 78, 1, 130-156.

Schotter, A. AND SopHer, B. 2003. Social learning and coordination conventions in intergenerational games: An experimental study. J. Political Econ. 111, 3.

Sмiтн, J. M. 1982. Evolution and the Theory of Games. Cambridge University Press, Cambridge, UK.

SzABó, G. AND TóKE, C. 1998. Evolutionary prisoner's dilemma game on a square lattice. Phys. Rev. E 58, 1, 69-73.

TAYlor, P. D. AND Jonker, L. B. 1978. Evolutionary stable strategies and game dynamics. Math. Biosci. 40, 1-2, 145 - 156 .

Traulsen, A. and Hauert, C. 2009. Stochastic evolutionary game dynamics. In Reviews of Nonlinear Dynamics and Complexity, H.-G. Shuster, Ed. Wiley-VHC, New York.

Traulsen, A., Pacheco, J. M., And Nowak, M. A. 2007. Pairwise comparison and selection temperature in evolutionary game dynamics. J. Theore. Biol. 246, 3, 522-529.

Tversky, A. AND Kahneman, D. 1992. Advances in prospect theory: Cumulative representation of uncertainty. J. Risk and Uncertainty 5, 297-323.

VEGa-Redondo, F. 1997. The evolution of walrasian behavior. Econometrica 65, 2, 375-384.

von Neumann, J. And Morgenstern, O. 1944. Theories of Games and Economic Behavior. Princeton University Press Princeton, NJ.

Received March 2010; revised June 2010; accepted June 2010 